\title{
A Case Report of Cemento-Ossifying Fibroma
}

\author{
Hashmi G. Sarwar $\cdot$ M. K. Jindal $\cdot$ Samshad Ahmad
}

Received: 10 October 2007/ Accepted: 10 March 2010/Published online: 22 September 2010

(C) Association of Oral and Maxillofacial Surgeons of India 2010

\begin{abstract}
The concept of 'fibro-osseous lesions' of bone evolved over the last several decades to include two major entities: fibrous dysplasia and ossifying fibroma as well as the other less common lesions such as florid osseous dysplasia, periapical dysplasia, focal sclerosing osteomyelitis, proliferative periostitis of garrie and ostitis deformans. The cemento-ossifying fibroma is a central neoplasm of bone as well as periodontium which has caused considerable controversy because of confusion of terminology and criteria of diagnosis. The cemento-ossifying fibroma is odontogenic in origin where as ossifying fibroma of bony origin. This article reports a case of an 11-year-old male who came to us with the history of swelling at the maxillary anterior region causing difficulty in closing of mouth as well as mastication.
\end{abstract}

Keywords Cemento-ossifying fibroma .

Ossifying fibroma $\cdot$ Odontogenic tumour .

Fibro-osseous lesions · Maxilla

\section{Introduction}

Cemento-ossifying fibroma is a fibro-osseous lesion that arises from the periodontal membrane [1]. The periodontal

H. G. Sarwar $(\bowtie)$

Department of Oral and Maxillofacial Surgery, Faculty of Medicine, Z. A. Dental College, AMU, Aligarh 202002, India e-mail: sarwarhashmi@rediffmail.com

M. K. Jindal

Department of Pedodontics and Preventive Dentistry,

Z.A. Dental College, AMU, Aligarh, India

S. Ahmad

Department of Pathology, Z.A. Dental College, AMU,

Aligarh, India membrane is a layer of fibrous connective tissue surrounding the roots. It contains multipotential cells capable of forming cementum, lameller bone and fibrous tissue [2, 3]. Although central cemento-ossifying fibromas of the mandible are common, central cemento-ossifying fibroma of the maxilla are unusual tumours [4].

In 1872, Menzel gave the first description of a variant of ossifying fibroma called cemento-ossifying fibroma in a 35-year-old woman with a long standing large tumour of the mandible [5].

This lesion can be separated from fibrous dysplasia of bone and certain other fibro-osseous lesions which do not represent true neoplasms [6]. A close histogenetic relationship exists between the central cemento-ossifying fibroma and the central ossifying fibroma. They have stated that, based on the marked similarity between the two regarding predilection of age of occurrence, sex, race, location, roentgenographic appearance and clinical behaviour, these two lesions represent the same basic neoplastic process. The only difference is in the cell involved with its end product, cementum in one case, bone in the other.

\section{Clinical Features}

This tumour generally occurs in young and middle aged adults [7]. Marked predilection for occurrence in female by a ratio of 2:1, the mandible is commonly involved than the maxilla [8]. The lesion is generally asymptomatic until the growth produces a noticeable swelling and mild deformity, displacement of teeth may be the early clinical features.

\section{Roentgenographic Features}

In the early stages, the cemento-ossifying fibroma appears as a radiolucent lesion with no evidence of internal 
radiopacities. As the tumour apparently matures, there is increasing calcification, so that the radiolucent area becomes flecked with opacities until, ultimately the lesion appear as an extremely radiopaque mass. Displacement of adjacent teeth is common. One additional important diagnostic feature is the centrifugal growth pattern rather than a linear one, therefore the lesions grow by expansion equally in all direction and present as a round tumour mass.

\section{Histologic Features}

Histology consists of many delicate interlacing collagen fibres, seldom arranged in discrete bundles, interspersed by large numbers of active, proliferating fibroblast and cementoblast. As the lesion matures, the islands of cementum increase in number, enlarge and ultimately coalesce. This with the probable increase in degree of calcifications accounts for increasing radiopaqueness of the lesions on the roentgenograms.

\section{Treatment and Prognosis}

The lesion is sharply circumscribed and demarcated from bone and it should be excised conservatively. Recurrence is rare.

\section{Case Report}

An 11-year-old male child reported to the outpatient Department of Pedodontics and Preventive Dentistry, Faculty of Medicine, with complaining of inability to close the mouth as well as the inability to masticate the food since 6-8 months of age (Fig. 1). The patient was apparently asymptomatic 18 months back when he developed a small swelling at maxillary anterior labial region, which was gradually increasing in nature.

\section{Examination}

On examination, there was an uniform, well circumscribed, ovoidal growth present in the maxillary anterior region due to the patient was unable to close his mouth. The lesion showed the dimension of $3 \times 3 \mathrm{~cm}$. The overlying mucosa was coral pink in colour and firm in consistency. The texture was smooth and there was no compressibility and depressibility. Routine blood examination was done and the results obtained were $\mathrm{Hb} 11 \mathrm{~g} \%$, TLC 12,900/ccm, differential count consist of neutrophils $65 \%$, lymphocytes $27 \%$, eosinophils $8 \%$, basophiles and monocytes was nil in blood picture. The sedimentation rate was 24.0 at first hour,

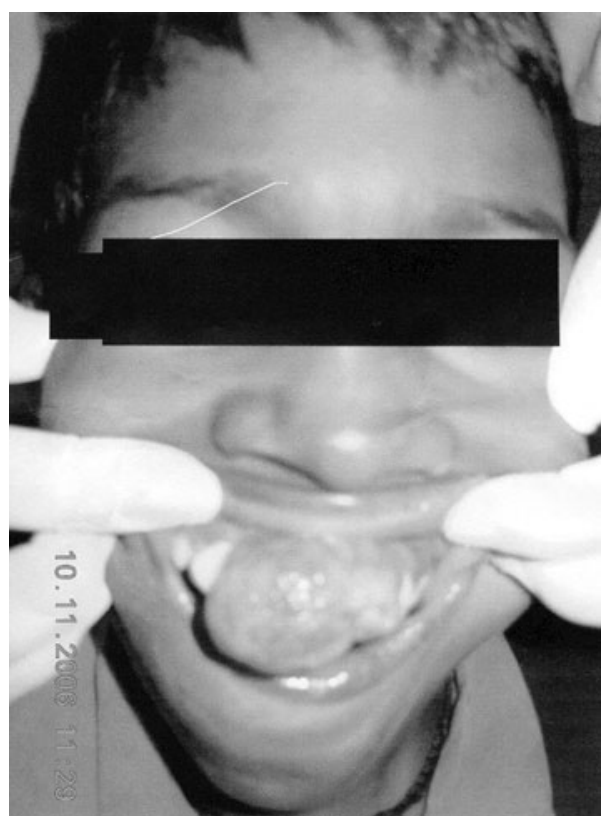

Fig. 1 Frontal pre operative view of the lesion

bleeding time (BT) and clotting time (CT) was 2.55 and $6.35 \mathrm{~min}$, respectively.

Radiographs

Occlusal view of maxilla (Fig. 2) was advised and radiographs revealed a well defined radiolucent area present between the permanent maxillary central incisor with displacement of both central incisors laterally, crown of incisors displaced towards canine area whereas root displaced towards midline. Left anterior region was flecked with multiple small radiopacities.

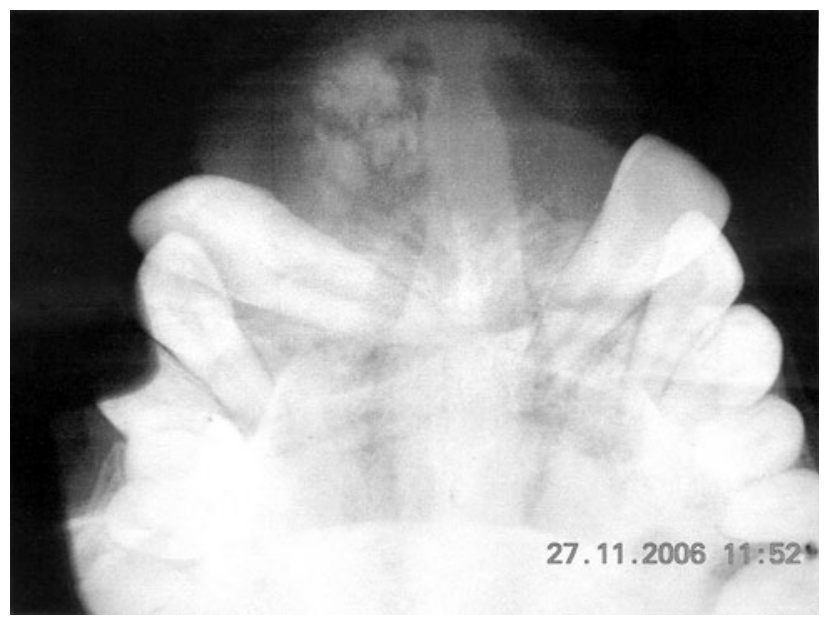

Fig. 2 Occlusal view of maxilla shows flecked radiopacities and flaring of the anterior teeth 


\section{Management}

$400 \mathrm{mg}$ Albendazole was advised 1 week before surgery to settle down the count of eosinophils. Preoperative antibiotics (amoxycillin $250 \mathrm{mg}, 1 \mathrm{tab}$ TDS) were advised for 3 days and excisional biopsy was planned. Surgical excision (Figs. 3 and 4) under local anaesthesia was done and after excision primary closure of the lesion was done with 3-0 silk sutures. After making two part of the excised mass, one part was sent to the Department of Pathology and second part was sent to the private laboratories to rule out the diagnostic confusion if any after histopathological examination. Antibiotic and anti-inflammatory drug regime was continued for the next 1 week. Alternate sutures were removed on the fifth postoperative days and complete sutures removal was done at seventh postoperative days. At this time the lesion showed signs of regression and patient was quite comfortable because now he able to eat without

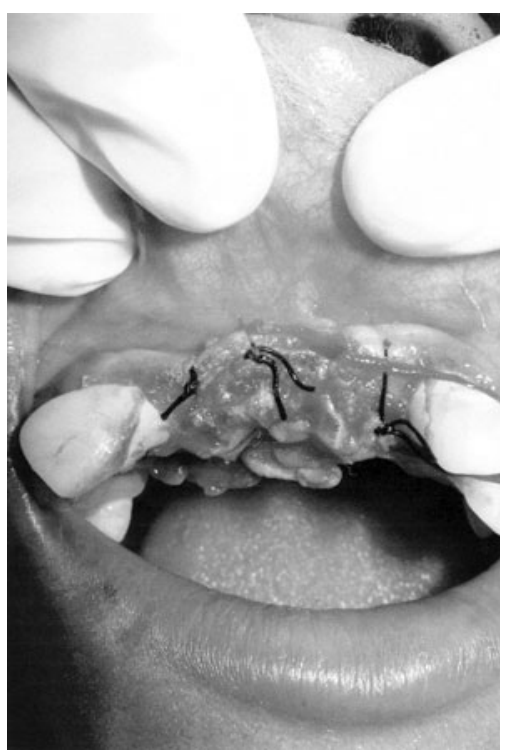

Fig. 3 Surgical area after excision

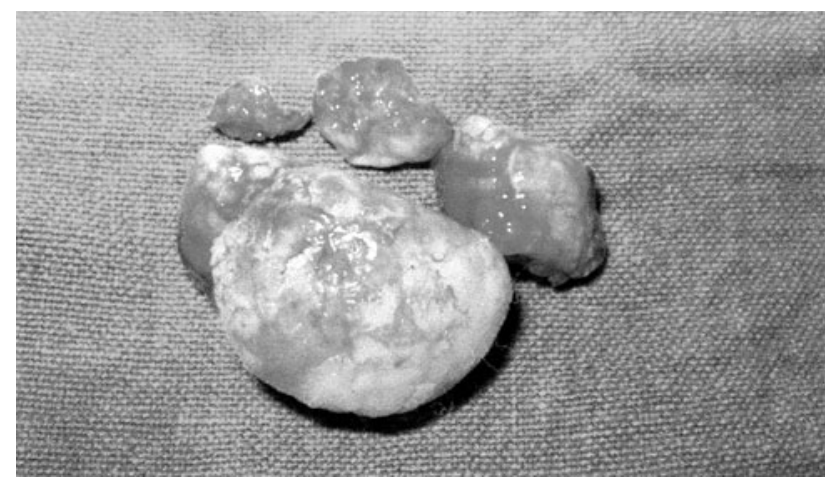

Fig. 4 Tumour mass immediately after excision

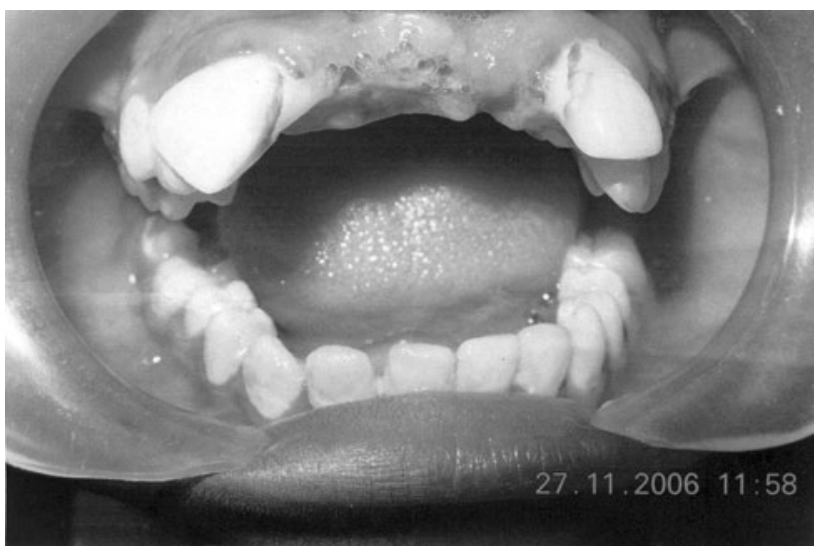

Fig. 5 Shows regressed lesion with normal edentulous ridge after two week of surgery

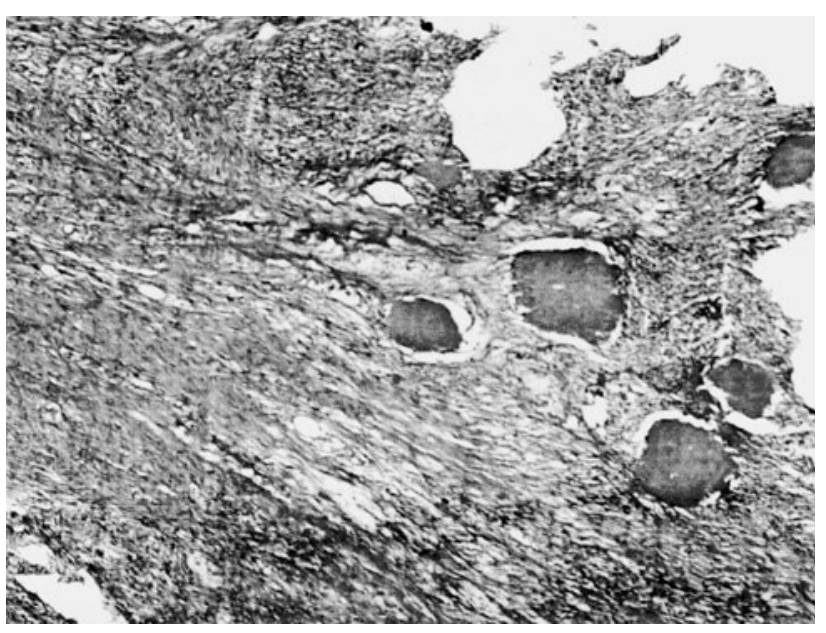

Fig. 6 Histopathological examination of the same lesion

any obstruction. Re-examination of the patient was done after 15 days and the lesion had regressed (Fig. 5), and the normal edentulous ridge was restored.

After post excisional biopsy the histopathological examination shows (Fig. 6) pseudoepitheliomatous hyperplasia of stratified squamous epithelium. At one edge, there is an ulcer with acute inflammation. The deeper areas show intense fibroblastic proliferation with mononuclear cell infiltrate with cementum and bony trabeculae are rimmed by osteoblast.

\section{Discussion}

Cemento-ossifying fibroma of the maxilla is an uncommon tumour. It is a well circumscribed tumour that grows exponentially and has clearly defined margins. Lesions are oval, spherical or multilocular and are clearly separated by surrounding bone and osteolytic borders [2]. 
Central cemento-ossifying fibromas are typically well defined, solitary radiolucencies with scattered foci. They maintain a spherical shape expand cortical bone without causing perforation and may cause divergence.

The neoplasm present an extremely variable radiographic appearance, depending upon its stage of development. However, despite the stage of development, the lesion is always well circumscribed and demarcated from surrounding bone, in contrast to true fibrous dysplasia. The central cementifying fibroma and its related lesion, the central ossifying fibroma, central cemento-ossifying fibroma, have a centrifugal growth pattern rather than a linear one. Therefore, lesions grow by expansion equally in all direction and present as round tumour masses.

Cemento-ossifying fibroma is a slow growing lesion composed of cellular fibroblastic tissue containing basophilic masses of cementum like tissue. In addition, varying amount of bony trabeculae are interspersed with in the lesion, giving it its characteristic appearance [8]. Reed [9] used the presence or absence of woven and lamellar bone in histopathological section to differentiate the cementoossifying fibroma from the other osseous lesions. Fibrous dysplasia contain no lamellar bone but rather, has a woven bone. On the other hand cemento-ossifying fibroma and the ossifying fibroma contain woven bone that is often rimmed by osteoblasts that have laid down layers of lamellar bone [10]. Additionally cemento-ossifying fibroma may have areas of cementum, appearing as psammoma bodies embedded in a benign fibrous stroma.

\section{Conclusion}

Benign odontogenic tumours

Odontogenic epithelium without odontogenic ectomesenchyme Ameloblastoma

Squamous odontogenic tumour (SOT)

Calcifying epithelial odontogenic tumour (CEOT, Pindborg tumour)

Odontogenic epithelium with odontogenic ectome-enchyme, with or without dental hard tissue formation

Ameloblastic fibroma (AF)

Ameloblastic fibrodentinoma (dentinoma)

Ameloblastic fibro-odontoma

Odontoameloblastoma

Adenomatoid odontogenic tumour (AOT)

Calcifying odontogenic cyst (COC)

Odontoma: complex and compound

Odontogenic ectomesenchyme with or without included

odontogenic epithelium

Odontogenic fibroma

Myxoma

Cementoblastoma
Malignant odontogenic tumours

Odontogenic carcinomas

Malignant ameloblastoma

Primary intraosseous carcinoma

Malignant variants of other odontogenic epithelial tumours

Malignant changes in odontogenic cysts

Odontogenic sarcomas

Ameloblastic fibrosarcoma (ameloblastic sarcoma)

Ameloblastic fibrodentinosarcoma and ameloblastic fibroodontosarcoma

Odontogenic carcinosarcoma

Neoplasms and other lesions related to bone osteogenic neoplasms

Cemento-ossifying fibroma (cementifying fibroma, ossifying

fibroma)

Nonneoplastic bone lesion

Fibrous dysplasia

Cemento-osseous dysplasias

Periapical cemental dysplasia (periapical fibrous dysplasia)

Florid cemento-osseous dysplasia

Other cemento-osseous dysplasias

Cherubism

Central giant cell granuloma

Aneurysmal bone cyst

Solitary bone cyst

\section{References}

1. Huebner GR, Brenneise CV, Ballenger J (1988) Central ossifying fibroma of the anterior maxilla: report of case. J Am Dent Assoc 116(4):507-510

2. Bertrand S, Eloy PH, Cornelis JP, Gosseye S, Clotuche J, Gilliard C (1993) Juvenile aggressive ossifying fibroma: case report and review of the literature. Laryngoscope 103(12):1385-1390

3. Hamner JE III, Lightbody PM, Ketcham AS, Swerdlow H (1968) Cemento-ossifying fibroma of the maxilla. Oral Surg Oral Med Oral Pathol 26(4):579-587

4. Sarita M, Raj KA, Daya SM, Rohtas KY (2000) Cemento-ossifying fibroma of the maxilla. Indian J Radiol Image 10(2): 103-104

5. Hamner JE III, Scofield HH, Cornyn J (1968) Benign fibroosseous jaw lesions of periodontal membrane origin: an analysis of 249 cases. Cancer 22(4):861-878

6. Waldron CA, Giansanti JS (1973) Benign fibro-osseous lesions of the jaw: a clinical-radiologic-histologic review of 65 cases. Oral Surg Oral Med Oral Pathol 35(2):190-201

7. Montgomery AH (1938) Ossifying fibroma of the jaw. Arch Surg 36:874-898

8. Kuta AJ, Macdonald W, Kaugars GE (1995) Central cementoossifying fibroma of the maxilla: a review of six cases. AJNR 16:1282-1286

9. Reed RJ (1963) Fibrous dysplasia of bone. A review of 25 cases. Arch Pathol 75:480-495

10. Spiet HJ, Dorfman HD, Fetchner RE, Ackerman LV (1971) Tumours of bone and cartilage in atlas of tumor pathology. Armed Forces Institute of Pathology: section II, face V 